\title{
Multidimensional Speckle Noise Model
}

\author{
Carlos López-Martínez \\ Equipe SAPHIR, Institut d'Electronique et de Télécommunications de Rennes UMR CNRS 6164, Université de Rennes 1, \\ Campus de Beaulieu, Building 11D, Room 101, 263 Avenue General Leclerc, 35042 Rennes Cedex, France \\ Email: carlos.lopez@univ-rennes1.fr
}

\section{Xavier Fàbregas}

Grup de Teledeteccio Activa, Departament de Teoria del Senyal i Communicacions, Universitat Politecnica de Catalunya (UPC), Campus Nord, Building D3, Room 118, Calle Jordi Girona 1-3, 08034 Barcelona, Spain

Email:fabregas@tsc.upc.es

\section{Eric Pottier}

Equipe SAPHIR, Institut d'Electronique et de Télécommunications de Rennes UMR CNRS 6164, Université de Rennes 1, Campus de Beaulieu, Building 11D, Room 101, 263 Avenue General Leclerc, 35042 Rennes Cedex, France

Email: eric.pottier@univ-rennes1.fr

Received 30 June 2004; Revised 23 November 2004

\begin{abstract}
One of the main problems of SAR imagery is the presence of speckle noise, originated by the inherent coherent nature of this type of systems. For one-dimensional SAR systems it has been demonstrated that speckle can be considered as a multiplicative noise term. Nevertheless, this simple model cannot be exported when multidimensional SAR imagery is addressed. This paper is devoted to present the latest advances into the definition of a multidimensional speckle noise model which does not depend on the data dimensionality. Speckle noise may be modeled by multiplicative and additive noise sources, whose combination is determined by the data's correlation structure. The validity of the proposed model is demonstrated by its application to a real L-band multidimensional SAR dataset acquired by the German ESAR sensor.
\end{abstract}

Keywords and phrases: multidimensional SAR imagery, speckle noise, noise modeling.

\section{INTRODUCTION}

Synthetic aperture radar (SAR) has become a well established, active, microwave imaging technique capable of monitoring, and characterizing, the surface of the Earth as well as its dynamics. In a first period, one-dimensional SAR systems allowed to demonstrate the capacities of this technology to provide information about the Earth surface reflectivity with a high spatial resolution, independently of the weather conditions or the day-night cycle [1]. But, the availability of multidimensional SAR systems which occurred in the last decade has been the fact which has really boosted the interest of the remote sensing community in these systems [2].

Multidimensional SAR systems open the possibility to increase the quantity of information which can be gathered from the scene under observation, and therefore, to better characterize it in a quantitative way. The additional

This is an open access article distributed under the Creative Commons Attribution License, which permits unrestricted use, distribution, and reproduction in any medium, provided the original work is properly cited. information that multichannel data sets provide arises through the increased parameter space of the acquisitions as well as the correlation structure between the channels. The associated literature is rich in examples showing the capacity of multidimensional SAR systems to perform quantitative remote sensing. A first example can be found in the so-called interferometric SAR (InSAR) systems [3], which allow obtaining the terrain's relief or even, variations of the topography by considering differential InSAR techniques $[4,5]$. Perhaps, the most relevant example of multidimensional SAR imagery is the one provided by polarimetric SAR systems (PolSAR) [6]. Since this SAR configuration, based on considering wave polarization diversity, is sensitive to the scattering properties of the target under study, it has been demonstrated as a useful tool to characterize quantitatively surface scattering $[7,8]$. In addition, the combination of the interferometric and the polarimetric capacities together into polarimetric SAR interferometry (PolInSAR), has permitted the study of vegetated areas [9]. Nevertheless, it must not be forgotten that there exist additional multidimensional SAR configurations based on different sources of diversity, 
such as SAR tomography [10], multitemporal or multifrequency SAR [11], which have demonstrated the potential of the multidimensional SAR data for quantitative remote sensing.

One of the main properties of the SAR systems is the capability to provide reflectivity information with a high spatial resolution. On the one hand, in the dimension perpendicular to the track of the platform carrying the SAR system, called range dimension, this spatial resolution is achieved by means of pulse compression techniques [1]. On the other hand, in the parallel dimension, denoted by azimuth, the high spatial resolution is obtained by a coherent recording and processing of the returned echoes. This coherent nature, which characterizes SAR systems, is also the origin of one of the most important problems of SAR imagery, namely, speckle noise $[12,13,14,15]$. Despite speckle consists of a true electromagnetic measurement, it must be considered as a noise component in order to assure access to the useful signal component $[14,16]$.

For a complete understanding of the speckle noise effects upon the useful signal component a noise model is required. Such a model is employed to identify, in the recorded data, the useful signal component, the noise term, or terms, and how they interact. In the case of one-dimensional SAR imagery, for homogenous areas, speckle is characterized by a multiplicative nature $[13,14]$. However, for multidimensional SAR data, the speckle noise present in a Hermitian product formed from a channel pair can no longer be modeled by a multiplicative noise source $[16,17]$. The aim of this paper is, hence, to present the latest advances on the definition and study of a general speckle noise model valid for any type of multidimensional SAR configuration.

The paper has been divided as follows. Section 2 introduces the formulation employed to describe multidimensional data. Section 3 describes the noise model for the phase component of a Hermitian product, which is extended to the complete Hermitian product in Section 4. This section contains also the corresponding extension to present the final multidimensional speckle noise model. The speckle noise model is analyzed in Section 5. Finally, Section 6 presents the main conclusions of this study.

\section{MULTIDIMENSIONAL SAR DATA REPRESENTATION}

A multidimensional SAR system, in what follows, will be considered as a system capable of recording $m$ different SAR images. Hence, the set of images is represented by the complex $m$-dimensional target vector

$$
\mathbf{k}=\left[S_{1}, S_{2}, \ldots, S_{m}\right]^{T},
$$

where $S_{i}$ for $i=1,2, \ldots, m$ represents each one of the complex SAR images, and $T$ denotes transposition. In (1), the subindex indicates that the $m$ SAR images have been acquired under some type of diversity, such as space or time diversity, wave polarization diversity, or frequency diversity. In the particular case of PolSAR data, (1) is derived from a vectorization process of the scattering matrix [18]. As demonstrated, this vector-based formulation allows to overcome the limitations of a matrix-based formulation in Pol$\operatorname{SAR}[18,19]$.

In the same way as (1) represents an extension of the idea of target vector derived for PolSAR data, one can also export the concepts of deterministic or point target and distributed target to a framework of multidimensional SAR data. On the one hand, the vector $\mathbf{k}$ is able to completely characterize a point target, that is, a target whose properties can be directly retrieved from $\mathbf{k}$ [18]. On the other hand, for distributed scatterers, the target vector $\mathbf{k}$ can no longer be considered to be a deterministic quantity, rather it may be modeled as an $m$-dimensional random variable $[18,20]$. Based on the coherent nature of SAR systems, under the Gaussian scattering assumption, and by considering the central limit theorem, $\mathbf{k}$ must be supposed to be described, for homogeneous areas, by a zero-mean, multidimensional, complex Gaussian random variable with probability density function (pdf) [21,22]

$$
p_{\mathbf{k}}(\mathbf{k})=\frac{1}{\pi^{m}|\mathbf{C}|} \exp \left(-\mathbf{k}^{H} \mathbf{C}^{-1} \mathbf{k}\right),
$$

where $H$ denotes transpose complex conjugation. From (2), it is possible to deduce, by using statistical arguments, the impossibility of $\mathbf{k}$ to characterize data, since, its average value is equal to $\mathbf{0}$. The pdf given by (2) is completely described by the $m \times m$, positive semidefinite, Hermitian covariance matrix $\mathbf{C}$,

$$
\mathbf{C}=E\left\{\mathbf{k k}^{H}\right\}=\left[\begin{array}{cccc}
E\left\{S_{1} S_{1}^{H}\right\} & E\left\{S_{1} S_{2}^{H}\right\} & \cdots & E\left\{S_{1} S_{m}^{H}\right\} \\
E\left\{S_{2} S_{1}^{H}\right\} & E\left\{S_{2} S_{2}^{H}\right\} & \cdots & E\left\{S_{2} S_{m}^{H}\right\} \\
\vdots & \vdots & \ddots & \vdots \\
E\left\{S_{m} S_{1}^{H}\right\} & E\left\{S_{m} S_{2}^{H}\right\} & \cdots & E\left\{S_{m} S_{m}^{H}\right\}
\end{array}\right]
$$

where $E\{x\}$ represents the expectation operator. Consequently, since the covariance matrix $\mathbf{C}$ determines the statistical behavior of $\mathbf{k}$ for distributed scatterers, it is capable of completely characterizing them [18].

The covariance matrix $\mathbf{C}$ needs to be estimated from the recorded $m$-dimensional target vector $\mathbf{k}$. By assuming statistical ergodicity and homogeneity, the expectation operator in (3) can be substituted by a spatial averaging, called multilook, which allows defining the so-called sample covariance matrix

$$
\mathbf{Z}_{n}=\frac{1}{n} \sum_{i=1}^{n} \mathbf{k}_{i} \mathbf{k}_{i}^{H} .
$$

In (4), $n$ denotes the number of samples which have been employed to estimate $\mathbf{C}$. In the following, the symbol $\mathbf{Z}$, will denote the one-look sample covariance matrix for $n=1$. Indeed, the sample covariance matrix consists of the maximum likelihood estimator of $\mathbf{C}$ [16]. Since the multidimensional variable $\mathbf{Z}_{n}$ is a random variable, the knowledge of its corresponding statistics are of primary interest, especially for the one-look data case, that is, $\mathbf{Z}$. 
Considering the Jacobian associated with the change of variables given by (4) into (2), one can demonstrate that the sample covariance matrix is characterized by the classical Wishart distribution $[21,22,23]$

$$
p_{\mathbf{Z}_{n}}\left(\mathbf{Z}_{n}\right)=\frac{n^{m n}\left|\mathbf{Z}_{n}\right|^{n-m}}{|\mathbf{C}|^{n} \tilde{\Gamma}_{m}(n)} \operatorname{etr}\left(-n \mathbf{C}^{-1} \mathbf{Z}_{n}\right)
$$

where $\operatorname{tr}(\mathbf{X})$ is the exponential of the trace of the matrix $\mathbf{X}$, and the multivariate gamma function is defined as follows:

$$
\tilde{\Gamma}_{m}(n)=\pi^{m(m-1) / 2} \prod_{i=1}^{m} \Gamma(n-i+1) .
$$

The Wishart distribution, as defined in (5), must be considered with certain restrictions, since the matrices $\mathbf{C}$ and $\mathbf{Z}_{n}$ have to be positive definite, that is, of rank $m$ [24]. This fact imposes that the expression of the Wishart distribution given by (5) is only valid for $n \geq m$. Therefore, it cannot be considered for multidimensional single-look SAR data characterization. Recently, an extension of (5), taking into account those cases in which $0<n<m$ has been presented in [25]. The extension considers the Wishart distribution as the set of probabilities on the closed cone of nonnegative matrices, denoted by $\bar{\Omega}$, of the space of Hermitian matrices generated by the measure $\mu_{p}$ :

$$
p_{\mathbf{Z}_{n}}\left(\mathbf{Z}_{n}\right)\left(d \mathbf{Z}_{n}\right)=\frac{1}{|\mathbf{C}|^{n}} e^{-\left\langle\mathbf{C}^{-1}, \mathbf{Z}_{n}\right\rangle} \mu_{p}\left(d \mathbf{Z}_{n}\right)
$$

where $d \mathbf{Z}_{n}$ denotes the differential volume on $\bar{\Omega}$ and $\langle\cdot, \cdot\rangle$ represents the inner product. When $n \geq m$, (7) reduces to (5) [25]. As observed, the generalized Wishart distribution (7) is defined in a differential form and in terms of the measure $\mu_{p}$, making its practical use extremely complicated. Thus, a different approach is necessary for the study of the statistics of $\mathbf{Z}$.

The one-look sample covariance matrix $\mathbf{Z}$, as given by (5), depends on $m$, and on the covariance matrix C. In addition, a multidimensional speckle noise model is understood as a multivariate function of the type

$$
\mathbf{Z}=f\left(\mathbf{C}, n_{1}, n_{2}, \ldots, n_{p}\right) .
$$

Equation (8) has to be able to identify, within the one-look sample covariance matrix $\mathbf{Z}$, the useful signal component, that is, $\mathbf{C}$, and how this component is corrupted by the speckle noise component, or components, indicated in (8) by $n_{i}$ for $i=1,2, \ldots, p$.

The following section of this paper is focused on deriving a multidimensional speckle noise model, as given by (8), to characterize the sample covariance matrix $\mathbf{Z}$.

\subsection{SAR images Hermitian product}

The Hermitian product of a pair of SAR images, that is,

$$
S_{i} S_{j}^{*}, \quad i, j=1,2, \ldots, m,
$$

where $*$ denotes complex conjugation, is a key quantity since it represents, as observed in (4), the basic building block when multidimensional SAR imagery is addressed. As it has been stated, the Hermitian product for distributed scatterers has a random nature. This randomness depends on the complex correlation coefficient

$\rho=|\rho| \exp \left(j \phi_{x}\right)=\frac{E\left\{S_{i} S_{j}^{*}\right\}}{\sqrt{E\left\{\left|S_{i}\right|^{2}\right\} E\left\{\left|S_{j}\right|^{2}\right\}}}, \quad i, j=1,2, \ldots, m$.

The amplitude of the complex correlation coefficient, that is, $|\rho|$, is called coherence, whereas $\phi_{x}$ refers to the average phase difference. In what follows the average power in the two channels is denoted as $\psi=\sqrt{E\left\{\left|S_{i}\right|^{2}\right\} E\left\{\left|S_{j}\right|^{2}\right\}}$ for $i, j=1,2, \ldots, m$.

The Hermitian product in (9) can be decomposed as

$$
\begin{aligned}
S_{i} S_{j}^{*} & =\left|S_{i} S_{j}^{*}\right| \exp \left(j\left(\phi_{i}-\phi_{j}\right)\right) \\
& =z \exp (j \phi), \quad i, j=1,2, \ldots, m,
\end{aligned}
$$

where $z$ is its amplitude and $\phi$ denotes the measured phase difference. The analysis to obtain the multidimensional speckle noise model will be based on a down-up approach. This method, first, considers the speckle noise model for a general Hermitian product of a pair of SAR images, based on the statistics of its different components [22]. Then, the results are extended to a multidimensional speckle noise model for the one-look sample covariance matrix $\mathbf{Z}$.

\section{PHASE DIFFERENCE NOISE MODEL}

From the statistics of the Hermitian product of a pair of SAR images, the argument of the $n$-look Hermitian product of two SAR images is described by the distribution [21]

$$
\begin{aligned}
p_{\phi}(\phi)= & \frac{\Gamma(n+1 / 2)\left(1-|\rho|^{2}\right)^{n} \beta}{2 \sqrt{\pi} \Gamma(n)\left(1-\beta^{2}\right)^{n+1 / 2}} \\
& +\frac{\left(1-|\rho|^{2}\right)^{n}}{2 \pi}{ }_{2} F_{1}\left(n, 1 ; \frac{1}{2} ; \beta^{2}\right),
\end{aligned}
$$

where $\beta=|\rho| \cos \left(\phi-\phi_{x}\right)$ and ${ }_{2} F_{1}(a, b ; c ; z)$ represents the Gauss hypergeometric function. The phase distribution in (12) presents its maximum at the value $\phi_{x}$. For instance, in interferometric applications, this phase contains terrain's relief data [3], whereas, for PolSAR data, it has been shown to contain relevant information about the scatterer being imaged [26].

Within the real phase interval $\left[\phi_{x}-\pi, \phi_{x}+\pi\right)$, the measured Hermitian product phase difference $\phi$ can be described by an additive noise model [27]

$$
\phi=\phi_{x}+v,
$$




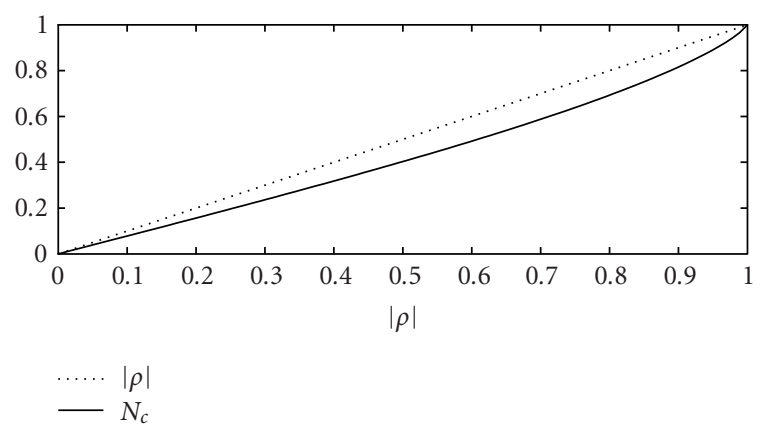

Figure 1: Parameter $N_{c}$ as a function of the coherence value $|\rho|$.

where $\phi_{x}$ denotes the true phase difference defined in (10), and $v$ is a zero-mean noise term, depending on $|\rho|$, and independent from $\phi_{x}$. In the following (13) is considered in the complex plane

$$
\exp (j \phi)=\cos (\phi)+j \sin (\phi) .
$$

Since a random variable can be separated into its mean value and a zero-mean random variable, hence, it is possible to define $\cos (v)=N_{c}+v_{1}^{\prime}$ and $\sin (v)=v_{2}^{\prime}$, which if introduced into (14) allows us to write

$$
\begin{aligned}
& \cos (\phi)=N_{c} \cos \left(\phi_{x}\right)+v_{1}^{\prime} \cos \left(\phi_{x}\right)-v_{2}^{\prime} \sin \left(\phi_{x}\right), \\
& \sin (\phi)=N_{c} \sin \left(\phi_{x}\right)+v_{1}^{\prime} \sin \left(\phi_{x}\right)+v_{2}^{\prime} \cos \left(\phi_{x}\right) .
\end{aligned}
$$

The parameter $N_{c}$, which corresponds to $E\{\cos (v)\}$, is determined, for one-look SAR imagery, that is, $n=1$, by

$$
N_{c}=\frac{\pi}{4}|\rho|_{2} F_{1}\left(\frac{1}{2}, \frac{1}{2} ; 2 ;|\rho|^{2}\right),
$$

where, as deduced from Figure 1, it contains the same information as the coherence value $|\rho|$. In [28], the authors have employed $N_{c}$ to introduce a novel coherence estimator. In addition, the terms $v_{1}^{\prime}$ and $v_{2}^{\prime}$, present in (15), are two noise terms such that

$$
\begin{aligned}
& E\left\{v_{1}^{\prime}\right\}=0, \quad \operatorname{var}\left\{v_{1}^{\prime}\right\} \cong \frac{1}{2}\left(1-|\rho|^{2}\right)^{0.79}, \\
& E\left\{v_{2}^{\prime}\right\}=0, \quad \operatorname{var}\left\{v_{2}^{\prime}\right\} \cong \frac{1}{2}\left(1-|\rho|^{2}\right)^{0.58} .
\end{aligned}
$$

In (17), the variance values represent approximations of the true values, since the corresponding analytical expressions prevent the extraction of any conclusion concerning the behavior of these noise terms due to its complexity [29]. Considering $E\left\{v_{1}^{\prime} v_{2}^{\prime}\right\}=0$, (15) can be simplified to

$$
\begin{aligned}
& \cos (\phi)=N_{c} \cos \left(\phi_{x}\right)+v_{c}, \\
& \sin (\phi)=N_{c} \sin \left(\phi_{x}\right)+v_{s},
\end{aligned}
$$

where

$$
\begin{gathered}
E\left\{v_{c}\right\}=E\left\{v_{s}\right\}=0, \\
\operatorname{var}\left\{v_{c}\right\}=\operatorname{var}\left\{v_{s}\right\} \cong \frac{1}{2}\left(1-|\rho|^{2}\right)^{0.685} .
\end{gathered}
$$

Thus, the complex phasor given at (14) can be described by the following noise model [29]:

$$
\exp (j \phi)=N_{c} \exp \left(j \phi_{x}\right)+\left(v_{c}+j v_{s}\right) .
$$

As observed in the previous equation, the first additive term contains the true phase information, that is, $\phi_{x}$, as would be expected. Nevertheless, this term also contains the information of coherence through the parameter $N_{c}$. This term is corrupted by the zero-mean, complex, additive term $v_{c}+j v_{s}$.

\section{MULTIDIMENSIONAL SPECKLE NOISE MODEL}

\subsection{Hermitian product noise model}

In this section, the speckle noise model for the Hermitian product argument, (21), is employed as the basis to derive a speckle noise model for the Hermitian product in (11). Thus, introducing the intermediate expressions of (15) into (11), the real and the imaginary parts of the Hermitian product take the form

$$
\begin{aligned}
& \mathfrak{R}\{z \exp (j \phi)\}=N_{c} z \cos \left(\phi_{x}\right)+z v_{1}^{\prime} \cos \left(\phi_{x}\right)-z v_{2}^{\prime} \sin \left(\phi_{x}\right), \\
& \mathfrak{I}\{z \exp (j \phi)\}=N_{c} z \sin \left(\phi_{x}\right)+z v_{1}^{\prime} \sin \left(\phi_{x}\right)+z v_{2}^{\prime} \cos \left(\phi_{x}\right),
\end{aligned}
$$

where $\mathfrak{R}\{x\}$ and $\mathfrak{I}\{x\}$ represent the real and imaginary parts of $x$, respectively. As observed, (22) can be decomposed into three additive terms, which statistical analysis allows, in the following, to obtain the speckle noise model for the Hermitian product of two SAR images. The interested reader is directed to [30] for the technical details of this process.

For homogeneous data, the statistics of the first additive terms of (22) are determined by the amplitude component $z$, since $N_{c}$ and $\phi_{x}$ correspond to constant values. As it can be observed from Figure 2, a direct relation cannot be established between the mean and the standard deviation values of $z$; the main consequence of which is the impossibility to introduce a noise source able to reproduce this behavior. The parameter $N_{c}$, introduced in Section 3 as a quantity similar to the coherence $|\rho|$, allows, nevertheless, to identify a noise mechanism with a multiplicative nature. As Figure 2 details, the effect of $N_{c}$ over the statistics of $z$, without considering power effects, is to transform it in such a way that the mean and the standard deviation present very similar values. This mechanism may be easily modeled by a homogeneous multiplicative noise source, denoted in the following as $n_{m}$. Hence,

$$
\begin{aligned}
z N_{c} \exp \left(j \phi_{x}\right) & \cong N_{c} E\{z\} n_{m} \exp \left(j \phi_{x}\right) \\
& =\psi \bar{z}_{n} n_{m} N_{c} \exp \left(j \phi_{x}\right),
\end{aligned}
$$




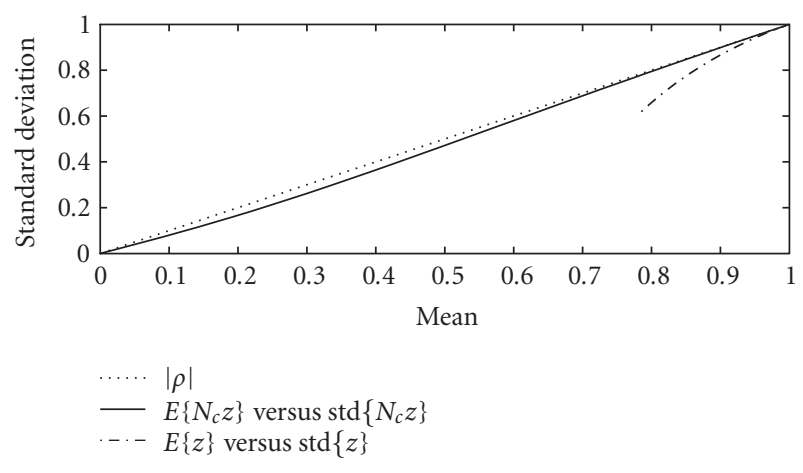

FIgUre 2: Mean versus standard deviation relations. This plot shows the influence of $N_{c}$ allowing to introduce a multiplicative noise mechanism.

where $E\{z\}=\psi \bar{z}_{n}$ has been considered and

$$
\bar{z}=\frac{\pi}{4}{ }_{2} F_{1}\left(-\frac{1}{2},-\frac{1}{2} ; 1 ;|\rho|^{2}\right) .
$$

It is important to observe that $N_{c}$ modulates the influence on the multiplicative speckle noise source $n_{m}$, in such a way that for low coherences, the effect of this noise source could be considered negligible. Finally, as concluded from (23), the multiplicative noise source $n_{m}$ corrupts only the amplitude information of the Hermitian product of two SAR images. This demonstrates that $n_{m}$ cannot explain the phase variability of the Hermitian product phase, that is, a multiplicative speckle noise model cannot be employed to characterize speckle noise for the real and imaginary parts of the Hermitian product of two SAR images.

Unlike the first additive terms of (22), the second ones are determined, from a statistical point of view, by the amplitude $z$ and the phase noise component $v_{1}^{\prime}$ introduced in Section 3. Fixing $\psi=1$ and $\cos \left(\phi_{x}\right)=1$, then $E\left\{z v_{1}^{\prime} \cos \left(\phi_{x}\right)\right\} \leq 0.2$ and $E\left\{z v_{1}^{\prime} \sin \left(\phi_{x}\right)\right\} \leq 0.2$, which determines that most of the mean value of the real and imaginary parts of the Hermitian product are concentrated in the first additive terms of (22). In order to determine the speckle noise source of the second additive terms, they are separated into the mean value and an additional zero-mean random variable, denoted as $n_{a 1}$ in the following. Consequently this new zero-mean random term may be considered as an additive speckle noise source. The variance properties of $n_{a 1}$ can be obtained from the distribution of the product $z v_{1}^{\prime}$. Nevertheless, the analytical expression of the variance presents such a complex equation, that it prevents from extracting any conclusion with respect to its dependence on the different signal parameters, and specially the coherence $|\rho|$. Thus, the next approximation is considered:

$$
\operatorname{var}\left\{z v_{1}^{\prime}\right\}=\psi^{2} \operatorname{var}\left\{n_{a 1}\right\}=\frac{1}{2} \psi^{2}\left(1-|\rho|^{2}\right)^{1.64},
$$

where its comparison with the actual variance values is given by Figure 3. The main characteristic of the speckle noise

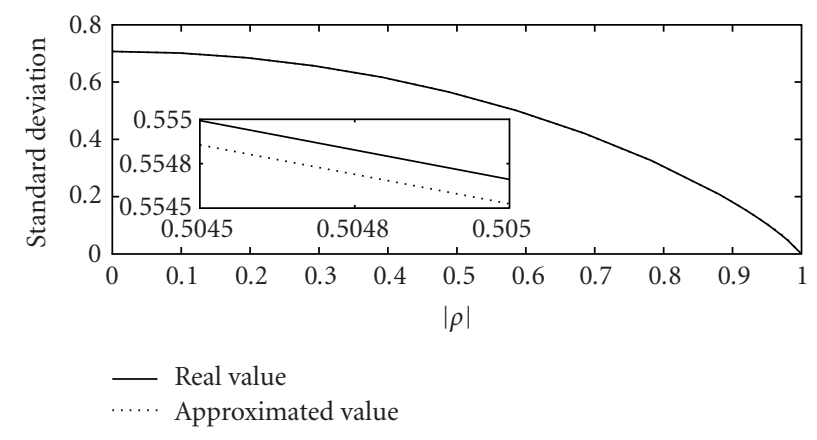

FIGURE 3: Real and approximated values for the standard deviation of the second additive term (25).

component $n_{a 1}$ is its dependence on $|\rho|$, giving as a result a nonhomogeneous speckle noise component. Introducing (25) into the second additive terms of (22), one can write

$$
z v_{1}^{\prime} \exp \left(j \phi_{x}\right)=\psi \exp \left(j \phi_{x}\right)\left\{\left(|\rho|-N_{c} \bar{z}_{n}\right)+n_{a 1}\right\}
$$

The additive speckle component will present its maximum variance for a null coherence. If one compares (26) with (23), it is possible to observe, from a qualitatively point of view that, without taking into account the modulation performed by the phase $\phi_{x}$, the real and imaginary parts of the Hermitian product of two SAR images are dominated by the multiplicative speckle component for high coherences, whereas they are dominated by the additive speckle term for lower ones.

Finally, the third additive terms of (22) can be analyzed in the same form presented in the previous paragraph. These terms depend statistically on the product $z v_{2}^{\prime}$. Considering the distribution of the real and imaginary parts of the Hermitian product of two SAR images, one can easily deduce that their mean value of $z v_{2}^{\prime}$ equals zero. Therefore, since the terms do not contain information, in terms of mean value, it can be considered that they are characterized by an additive noise term, which will be denoted as $n_{a 2}$. The variance of this term equals

$$
\operatorname{var}\left\{z v_{2}^{\prime}\right\}=\psi^{2} \operatorname{var}\left\{n_{a 2}\right\}=\frac{1}{2} \psi^{2}\left(1-|\rho|^{2}\right)
$$

which presents the same dependence on $|\rho|$ as $n_{a 1}$, see (25) and Figure 4. Similarly as performed in the previous paragraph, but considering the zero-mean value of the third additive terms of (22),

$$
j z v_{2}^{\prime} \exp \left(j \phi_{x}\right)=j \psi \exp \left(j \phi_{x}\right) n_{a 2}
$$

Consequently, a novel speckle noise term has been identified. If (28) is compared with (26), it can be deduced that $n_{a 1}$ and $n_{a 2}$, despite being in quadrature, present the same characteristics. 
Introducing (23), (26), and (28) into (22),

$$
\begin{aligned}
S_{i} S_{j}^{*}= & \psi \bar{z}_{n} n_{m} N_{c} \exp \left(j \phi_{x}\right) \\
& +\psi \exp \left(j \phi_{x}\right)\left\{\left(|\rho|-N_{c} \bar{z}_{n}\right)+n_{a 1}\right\}+j \psi \exp \left(j \phi_{x}\right) n_{a 2} \\
= & \psi \bar{z}_{n} n_{m} N_{c} \exp \left(j \phi_{x}\right)+\psi\left(|\rho|-N_{c} \bar{z}_{n}\right) \exp \left(j \phi_{x}\right) \\
& +\psi\left(n_{a 1}+j n_{a 2}\right) \exp \left(j \phi_{x}\right),
\end{aligned}
$$

which represents a first speckle noise model for the Hermitian product of two SAR images. Since the additive noise components $n_{a 1}$ and $n_{a 2}$ are uncorrelated, it is possible to simplify the last term of (29). Considering the corresponding real and imaginary parts, two novel additive speckle terms can be introduced as follows:

$$
\begin{aligned}
n_{a r} & =\cos \left(\phi_{x}\right) n_{a 1}-\sin \left(\phi_{x}\right) n_{a 2}, \\
n_{a i} & =\sin \left(\phi_{x}\right) n_{a 1}+\cos \left(\phi_{x}\right) n_{a 2} .
\end{aligned}
$$

From (30),

$$
E\left\{n_{a r}\right\}=0, \quad E\left\{n_{a i}\right\}=0 .
$$

With respect to the variance values of the additive speckle terms introduced within (30), it is clear that this value depends on the average phase $\phi_{x}$. As observed in Figure 4, the variance of the speckle terms $n_{a 1}$ and $n_{a 2}$ present such a similar value, that the dependence of $n_{a r}$ and $n_{a i}$ on $\phi_{x}$ can be eliminated by considering their variances to be equal to

$$
\operatorname{var}\left\{n_{a r}\right\}=\operatorname{var}\left\{n_{a i}\right\}=\frac{1}{2}\left(1-|\rho|^{2}\right)^{1.32} .
$$

The effect of this approximation is also depicted in Figure 4.

Then, as a final step, the definitions presented in (30) are introduced into (29). Consequently, the complex Hermitian product of two SAR images can be described by the following speckle noise model [30]:

$$
\begin{aligned}
S_{i} S_{j}^{*}= & \underbrace{\psi \bar{z}_{n} n_{m} N_{c} \exp \left(j \phi_{x}\right)}_{\text {Multiplicative term }} \\
& +\underbrace{\psi\left(|\rho|-N_{c} \bar{z}_{n}\right) \exp \left(j \phi_{x}\right)+\psi\left(n_{a r}+j n_{a i}\right)}_{\text {Additive term }} .
\end{aligned}
$$

The previous equation allows the identification of two speckle noise mechanisms. On the one hand, a real, homogeneous, multiplicative speckle term $n_{m}$, which only corrupts the amplitude of the Hermitian product. On the other hand, $n_{a r}+j n_{a i}$ represents a complex, nonhomogeneous, additive speckle term introducing noise both in amplitude and in phase.

\subsection{Multidimensional noise model formulation}

The complete analysis performed previously has allowed the derivation of a new speckle noise model for the Hermitian product of two SAR images. As it was made evident in Section 2, this product can be considered as the basic building block to completely characterize multidimensional SAR data under a covariance matrix-based formulation. Hence, the result presented above by (33) is now introduced into (4) (with $n=1$ ) in order to derive a multidimensional speckle noise model [31], in the philosophy presented by (8).

\subsubsection{Multidimensional multiplicative speckle noise component}

First, the multidimensional extension of the multiplicative term labeled into (33) is considered. As demonstrated in Section 4.1, the speckle term $n_{m}$ is homogeneous. Therefore, as it does not depend on any signal property, it appears in the diagonal, as well as, the off-diagonal elements of the one-look sample covariance matrix Z Z. Based on (33), and in particular (26), an $m \times m$, real matrix, containing the multiplicative speckle noise terms, is defined as

$$
\mathbf{N}_{m}=\left[\begin{array}{cccc}
n_{m}^{11} & n_{m}^{12} & \cdots & n_{m}^{1 m} \\
n_{m}^{21} & n_{m}^{22} & \cdots & n_{m}^{2 m} \\
\vdots & \vdots & \ddots & \vdots \\
n_{m}^{m 1} & n_{m}^{m 2} & \cdots & n_{m}^{m m}
\end{array}\right]
$$

whereas an $m \times m$, complex, Hermitian matrix containing the useful signal term is specified as

$$
\mathbf{C}_{m}=\left[\begin{array}{cccc}
\psi^{11} & \psi^{12} N_{c}^{12} \bar{z}_{n}^{12} e^{j \phi_{x}^{12}} & \cdots & \psi^{1 m} N_{c}^{1 m} \bar{z}_{n}^{1 m} e^{j \phi_{x}^{1 m}} \\
\psi^{21} N_{c}^{21} \bar{z}_{n}^{21} e^{j \phi_{x}^{21}} & \psi^{22} & \cdots & \psi^{2 m} N_{c}^{2 m} \bar{z}_{n}^{2 m} e^{j \phi_{x}^{2 m}} \\
\vdots & \vdots & \ddots & \vdots \\
\psi^{m 1} N_{c}^{m 1} \bar{z}_{n}^{m 1} e^{j \phi_{x}^{m 1}} & \psi^{2 m} N_{c}^{2 m} \bar{z}_{n}^{2 m} e^{j \phi_{x}^{2 m}} & \cdots & \psi^{m m}
\end{array}\right]
$$

It is important to highlight, that, the diagonal elements of the sample covariance matrix, (4), which are defined on the basis of the Hermitian product $S_{i} S_{i}^{*}$ for $i=1,2, \ldots, m$, can be considered to be characterized by a complex correlation 


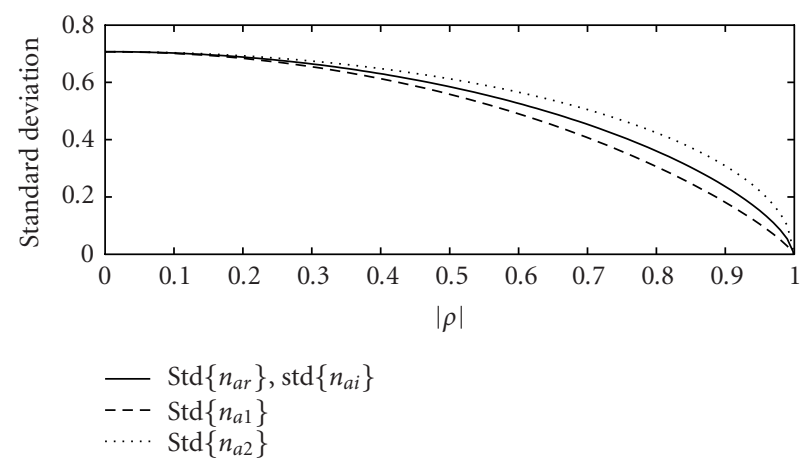

FIgURE 4: Standard deviation values for the different additive speckle noise terms identified for the Hermitian product of two SAR images.

coefficient equal to $1 \exp (j 0)$. Under this conditions, $N_{c}$ and $\bar{z}_{n}$ are equal to 1 , justifying the differences between the diagonal and the off-diagonal elements of (35).

When addressing the multidimensional extension of the multiplicative term, in order to maintain separately the noise term, (34), from the useful signal component, (35), a matrixbased formulation cannot be considered. Hence, a vectorbased formulation must be employed. As a result, the multiplicative term of (33) extends multidimensionally as

$$
\operatorname{diag}\left(\operatorname{vec}\left(\mathbf{N}_{m}\right)\right) \operatorname{vec}\left(\mathbf{C}_{m}\right)
$$

where vec $(\mathbf{X})$ represents an $m^{2} \times 1$ vector formed by staking the columns of $\mathbf{X}$, and $\operatorname{diag}(\mathbf{x})$ forms an $m^{2} \times m^{2}$ diagonal matrix with the elements of the vector $\mathbf{x}$.

\subsubsection{Multidimensional additive speckle noise component}

Although the additive term in (33) could be considered under a matrix-based formulation in the multidimensional extension, the vector-based formulation imposed by the multiplicative term, imposes, also, in this case, a vector-based formulation. Here, two new $m \times m$ matrices are defined. On the one hand, an $m \times m$, complex matrix, which contains the complex additive speckle noise terms:

$$
\mathbf{N}_{a}=\left[\begin{array}{cccc}
0 & \psi^{12}\left(n_{a r}^{12}+j n_{a i}^{12}\right) & \cdots & \psi^{1 m}\left(n_{a r}^{1 m}+j n_{a i}^{1 m}\right) \\
\psi^{21}\left(n_{a r}^{21}+j n_{a i}^{21}\right) & 0 & \cdots & \psi^{2 m}\left(n_{a r}^{2 m}+j n_{a i}^{2 m}\right) \\
\vdots & \vdots & \ddots & \vdots \\
\psi^{m 1}\left(n_{a r}^{m 1}+j n_{a i}^{m 1}\right) & \psi^{m 2}\left(n_{a r}^{m 2}+j n_{a i}^{m 2}\right) & \cdots & 0
\end{array}\right]
$$

And, on the other hand, an $m \times m$, complex matrix which

contains the useful signal component:

$$
\mathbf{C}_{a}=\left[\begin{array}{cccc}
0 & \psi^{12}\left(\left|\rho^{12}\right|-N_{c}^{12} \bar{z}_{n}^{12}\right) e^{j \phi_{x}^{12}} & \cdots & \psi^{1 m}\left(\left|\rho^{1 m}\right|-N_{c}^{1 m} \bar{z}_{n}^{1 m}\right) e^{j \phi_{x}^{1 m}} \\
\psi^{21}\left(\left|\rho^{21}\right|-N_{c}^{21} \bar{z}_{n}^{21}\right) e^{j \phi_{x}^{21}} & 0 & \cdots & \psi^{2 m}\left(\left|\rho^{2 m}\right|-N_{c}^{2 m} \bar{z}_{n}^{2 m}\right) e^{j \phi_{x}^{2 m}} \\
\vdots & \vdots & \ddots & \vdots \\
\psi^{m 1}\left(\left|\rho^{m 1}\right|-N_{c}^{m 1} \bar{z}_{n}^{m 1}\right) e^{j \phi_{x}^{m 1}} & \psi^{m 2}\left(\left|\rho^{m 2}\right|-N_{c}^{m 2} \bar{z}_{n}^{m 2}\right) e^{j \phi_{x}^{m 2}} & \cdots & 0
\end{array}\right] .
$$

It can be observed, from the previous two expressions, that the diagonal entries of the matrices are null. As noticed previously, the diagonal elements can be characterized by a complex correlation coefficient equal to $1 \exp (j 0)$. Introducing it into (32) for the additive noise components, and into (26) for the useful signal component, one can easily demonstrate the zeros at the diagonal of (37) and (38).

The additive component is, then, extended to a multidimensional framework, under the vector-based formulation 
imposed by the multiplicative one, as

$$
\operatorname{vec}\left(\mathbf{C}_{a}\right)+\operatorname{vec}\left(\mathbf{N}_{a}\right) .
$$

\subsubsection{Final multidimensional speckle noise model}

The final multidimensional speckle noise model for SAR data is derived by combining (36) and (39). Hence, the one-look covariance matrix can be written as [31]

$$
\operatorname{vec}(\mathbf{Z})=\operatorname{diag}\left(\operatorname{vec}\left(\mathbf{N}_{m}\right)\right) \operatorname{vec}\left(\mathbf{C}_{m}\right)+\operatorname{vec}\left(\mathbf{C}_{a}\right)+\operatorname{vec}\left(\mathbf{N}_{a}\right),
$$

where the vector-based formulation is imposed by the multiplicative speckle noise term in order to maintain separately the useful signal from the noise component. The main characteristic of (40) is that the multidimensional speckle noise model does not depend on the data dimensionality, that is, $m$. In (40), the useful signal component is encountered under

$$
\operatorname{vec}(\mathbf{C})=\operatorname{vec}\left(\mathbf{C}_{m}\right)+\operatorname{vec}\left(\mathbf{C}_{a}\right) \Longrightarrow \mathbf{C}=\mathbf{C}_{m}+\mathbf{C}_{a} .
$$

\subsection{Multidimensional speckle noise model characteristics}

First of all, (33) represents a generalization of the multiplicative speckle noise model developed for the SAR images intensity, since, as demonstrated, the diagonal elements of the one-look sample covariance matrix reduce to

$$
S_{i} S_{i}^{*}=\left|S_{i}\right|^{2}=\psi^{i i} n_{m}^{i i}=E\left\{\left|S_{i}\right|^{2}\right\} n_{m}^{i i}, \quad i=1,2, \ldots, m .
$$

In addition, (33) is also in accordance with the additive noise model for the phase difference, (13), by construction.

As (40) has made evident, the multidimensional speckle noise model presents a high degree of heterogeneity. As shown by (33), the speckle noise for a particular Hermitian product is determined by the combination of multiplicative and additive noise sources. This combination is controlled by the complex correlation coefficient which, additionally, varies among the elements of the covariance matrix. Consequently, the speckle noise is not equal for all the covariance matrix entries. This double source of heterogeneity shows the complexity of the speckle noise mechanism for multidimensional SAR data.

In the case of a particular element of the covariance matrix, attention must be paid to the fact that speckle depends on the complex correlation coefficient, that is, on the coherence $|\rho|$ and the phase $\phi_{x}$. As it can be deduced from (33), the coherence determines the importance of the different speckle noise sources. On the one hand, $|\rho|$ controls the weight of the multiplicative part of speckle through the modulation by $N_{c}$, (23). On the other hand, the variance of the speckle additive term depends on $|\rho|$ (32). As a result, low coherence areas of a Hermitian product are dominated by the additive term of speckle, whereas speckle for high coherence areas turns out to be dominated by the multiplicative term. Nevertheless, as one can observe in (33), the multiplicative term of speckle is also modulated by the phase term $\phi_{x}$. This second modulation has very important effects. First, the final nature of speckle noise for the real and imaginary parts of the Hermitian product of SAR images will differ, since the real part depends on $\cos \left(\phi_{x}\right)$, whereas the imaginary one depends on $\sin \left(\phi_{x}\right)$. Additionally, the phase dependence can provoke that, despite an area can be characterized by a high coherence, speckle noise may be dominated by an additive noise nature. It is clear that the phase of the complex correlation coefficient $\phi_{x}$ has an evident role in determining the final nature of speckle noise. This effect acquires a crucial importance in interferometric applications, since the dependence of $\phi_{x}$ on the terrain topography results in the fact that the topography has an enormous impact on determining the final speckle noise nature.

\section{EXAMPLES AND VALIDATION}

In what follows, the multidimensional speckle noise model is tested over real SAR data. This data correspond to an Lband, fully polarimetric PolInSAR dataset acquired by the German system ESAR, operated by DLR, over the region of Oberfapfenhoffen, Germany. The two interferometric acquisitions were collected in a repeat-pass configuration with an approximated baseline of 10 meters and a temporal difference of 10 minutes.

Due to the high dimensionality of the data, it is difficult to present the behavior of speckle noise for the complete dataset. However, since the multidimensional speckle noise model, (40), is based on the model for the Hermitian product of a pair of SAR images, it is sufficient to show the speckle behavior for the real or imaginary parts of a particular Hermitian product, extending, later, the derived results to the multidimensional data. From the presented dataset, the term $\mathfrak{R}\left\{S_{h h 1} S_{v v 2}^{*}\right\}$ is selected, where the SAR image, $S_{h h 1}$, corresponds to a first interferometric SAR image in which the signal is transmitted and received in the horizontal polarization. The second image, $S_{v v 2}$, is acquired, on the contrary, in a different spatial location where the signal is transmitted and received in the vertical polarization. The flat Earth term has been removed from data. Figure 5 presents the images corresponding to the complex correlation coefficient which characterizes this Hermitian product. If the mean and the standard deviation of $\mathfrak{R}\left\{S_{h h 1} S_{v v 2}^{*}\right\}$ are estimated over $7 \times 7$ pixels, nonoverlapping windows, and represented together, as given in Figure 6, one can deduce that speckle noise, that is, data variability, cannot be said to be determined, neither by a multiplicative nor by an additive speckle noise model [17].

The nature of speckle noise depends, first, on the coherence value. Hence, the data presented in Figure 5 are analyzed as a function of coherence. Thus, three coherence ranges are considered. In each case, the scatter diagram corresponding to the term $\mathfrak{R}\left\{S_{h h 1} S_{v v 2}^{*}\right\}$, as well as the ones corresponding to the multiplicative and the additive terms given in (33), have been derived. The multiplicative term is obtained by 


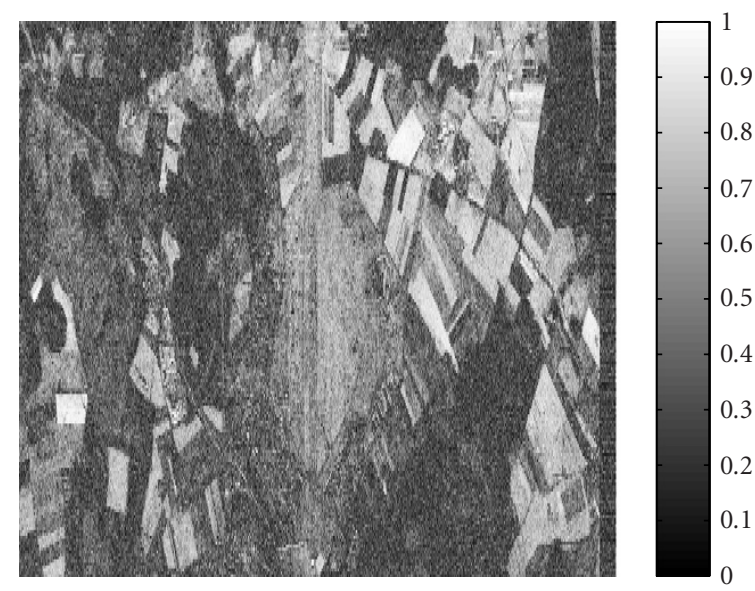

(a)

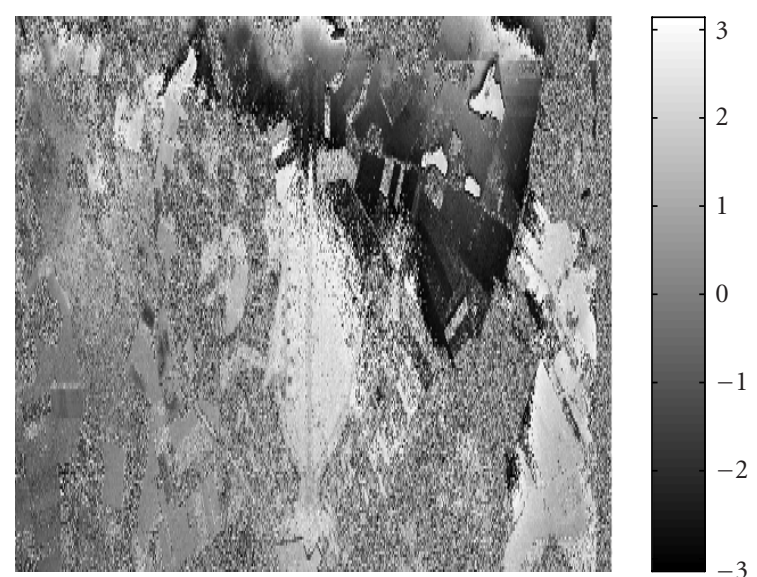

(b)

Figure 5: Complex correlation coefficient of the term $\mathfrak{R}\left\{S_{h h 1} S_{v v 2}^{*}\right\}$. (a) $|\rho|$. (b) $\phi_{x}$ in radians.

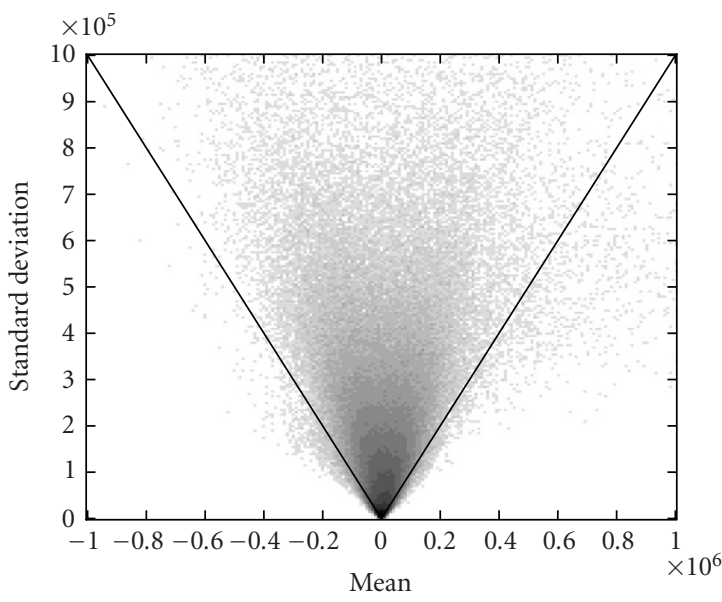

FIGURE 6: Scatter diagram of $\mathfrak{R}\left\{S_{h h 1} S_{v v 2}^{*}\right\}$.

multiplying $z$ by the term $N_{c} \exp \left(j \phi_{x}\right)$, obtained from $\rho$. The additive term is just calculated by subtracting the multiplicative term from the Hermitian product of two SAR images. The results are presented in Figure 7. As it can be deduced from Figure 7b, the higher the coherence value, the higher the importance of the multiplicative term of speckle. On the contrary, Figure $7 \mathrm{c}$ shows that the importance of the additive term of speckle decreases with increasing coherences. For low coherences, one can observe the similarity of the scatter diagram of $\mathfrak{R}\left\{S_{h h 1} S_{v v 2}^{*}\right\}$ with the one corresponding to the additive term. In this case, the signal has an average value close to zero, whereas the standard deviation can take any value. This fact confirms the additive nature of speckle noise in this case. On the contrary, for high coherences, the total scatter diagram for $\mathfrak{R}\left\{S_{h h 1} S_{v v 2}^{*}\right\}$ mimics the one corresponding to the one of the multiplicative term. Finally, for medium coherences, the total diagram, as observed, results from the combination of the two speckle noise terms.

As mentioned in Section 4.3, the final nature of speckle noise depends also on the phase term $\phi_{x}$, which has an important effect when interferometric applications are considered. This effect can be observed if PolSAR data is contrasted with PolInSAR data. Figure 8 compares PolSAR data, corresponding to $\mathfrak{R}\left\{S_{h h 1} S_{v v 1}^{*}\right\}$, and PolInSAR data, corresponding to $\mathfrak{R}\left\{S_{h h 1} S_{v v 2}^{*}\right\}$. Data correspond to three different homogeneous areas of relatively high coherence, about 0.8 , within the considered dataset. Since the coherence values are very similar in both datasets, it can be concluded that the three areas correspond to bare soil, where the sole difference is due to the topographic component. From Figure 8 it can be observed that the standard deviation of the additive component does not present differences neither between the PolSAR and PolInSAR data nor the three selected areas, confirming the fact that this speckle component does not depend on $\phi_{x}$. Nevertheless, the standard deviation curves corresponding to the multiplicative component of speckle, confirm, in the case of $\mathfrak{R}\left\{S_{h h 1} S_{v v 2}^{*}\right\}$, that the importance of this noise term is determined by the terrain's topography, which is not the case in case for $\mathfrak{R}\left\{S_{h h 1} S_{v v 1}^{*}\right\}$. From the first row of Figure 8 it can be observed that the decrease in value of the phase $\phi_{x}$ is translated into a decrease of the importance of the multiplicative speckle noise component, arriving to the extreme that the additive speckle noise term can dominate, even, for high coherences.

\section{CONCLUSIONS}

In the previous sections, a novel multidimensional SAR speckle noise model has been proposed and compared with experimental data. As demonstrated, the multidimensional noise model presents the property that it does not depend 

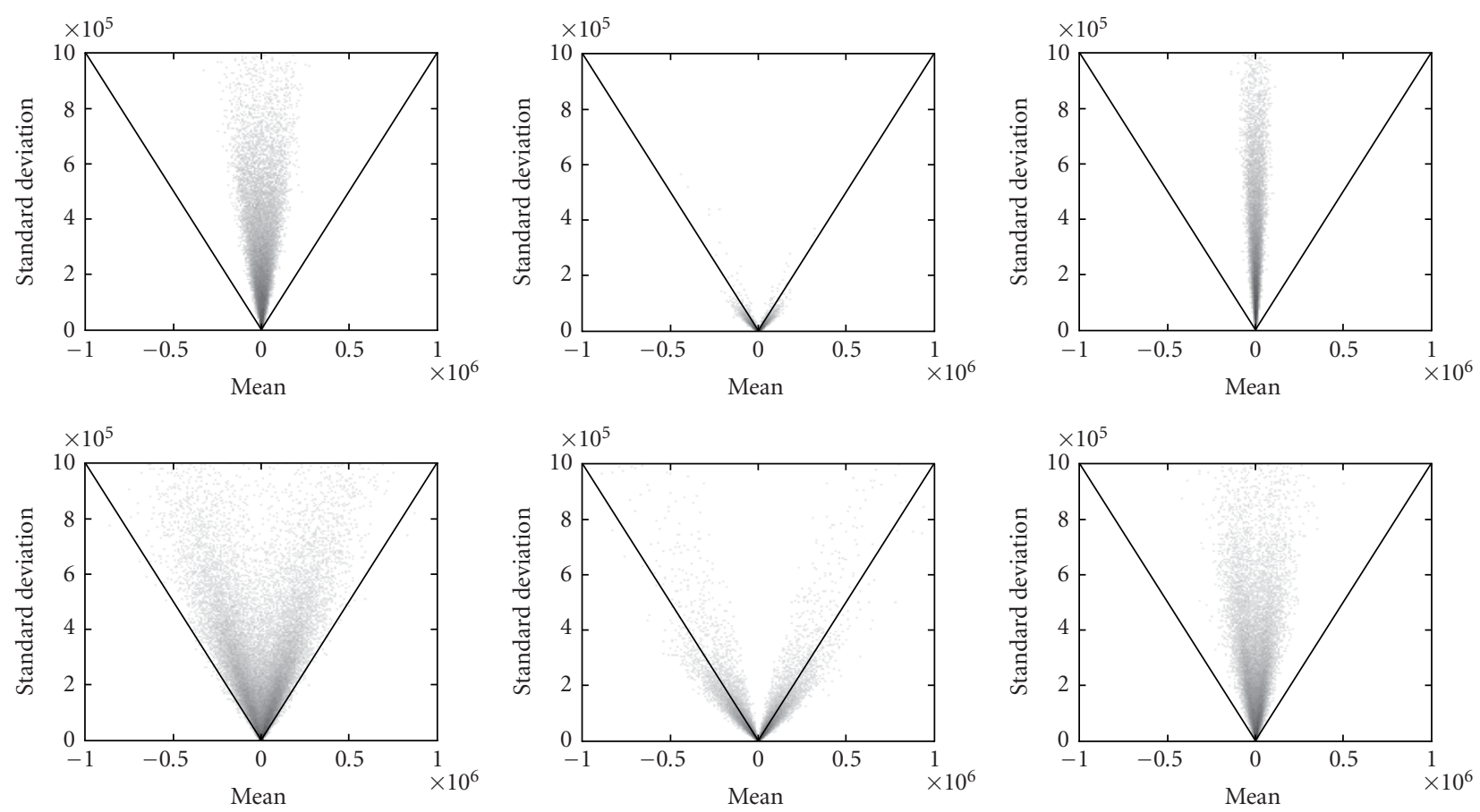

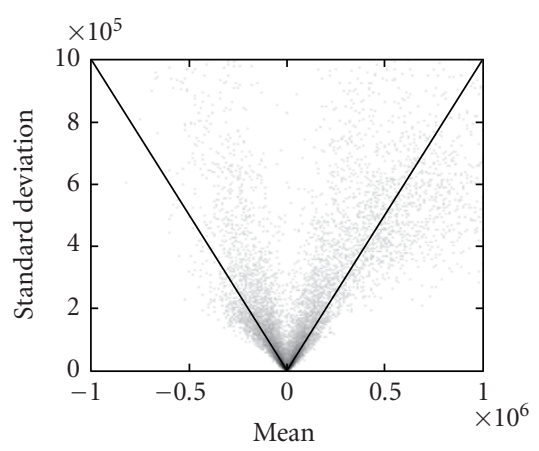

(a)

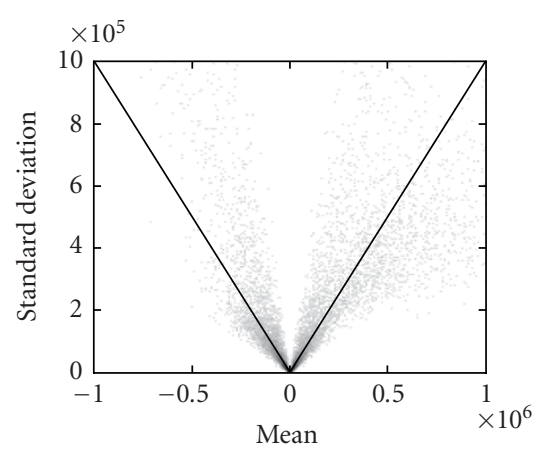

(b)

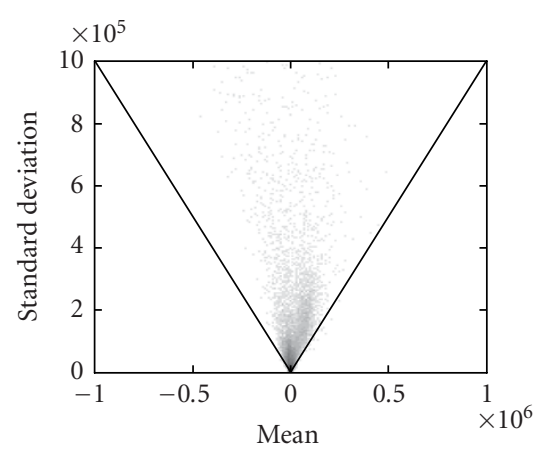

(c)

Figure 7: Scatter diagrams of the different terms of $\mathfrak{R}\left\{S_{h h 1} S_{v v 2}^{*}\right\}$ for all the coherence ranges. (a) Total. (b) Multiplicative noise term. (c) Additive noise term. The first row corresponds to $0 \leq|\rho| \leq 0.2$, the second row to $0.4 \leq|\rho| \leq 0.6$, and the third row to $0.8 \leq|\rho| \leq 1.0$.

neither on the data's dimensionality nor on the type of physical information they contain. This makes possible to apply the model to any type of multidimensional SAR imagery configuration.

First, a noise model for the complex phase of the Hermitian product of two SAR images has been derived. Then, this model has been extended to consider speckle noise within the complete Hermitian product. It is shown that, in this case, speckle noise may be modeled by the combination of a real, multiplicative noise term and a zero-mean, complex additive noise term. The final nature of speckle noise results from this combination which is determined by the complex correlation coefficient characterizing the Hermitian product. Since this product consists of the basic building block to construct the covariance matrix formulation, it has been employed to construct the multidimensional speckle model.
In this case, only a vector-based formulation is possible in order to maintain, both, the noise terms and the signal information content separately. The validity of the model has been confirmed by analyzing a real PolInSAR dataset. A final comparison with PolSAR data has demonstrated that speckle noise depends on the terrain's topography in the case of interferometric applications.

\section{ACKNOWLEDGMENTS}

The authors would kindly thank DLR for providing the SAR data employed in this study. This work has been financed by the RTN-AMPER project (HPRN-CT-2002-00205) and the Spanish MCYT-FEDER funds (TIC 2002-04451-C02-01). Finally, the authors would like to thank the anonymous reviewers for their useful comments. 


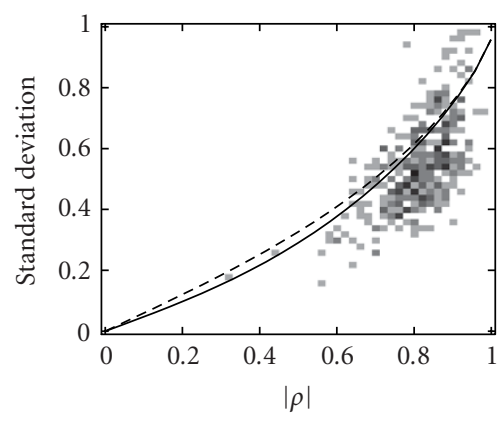

(a)

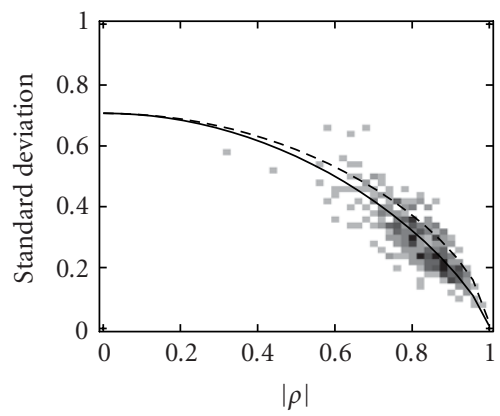

(d)

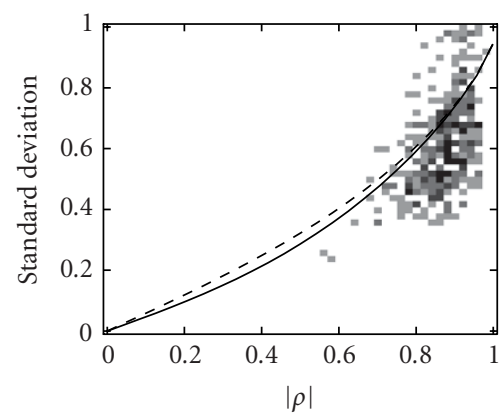

(g)

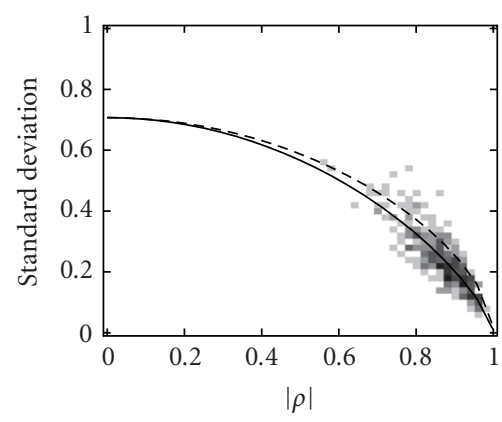

(j)

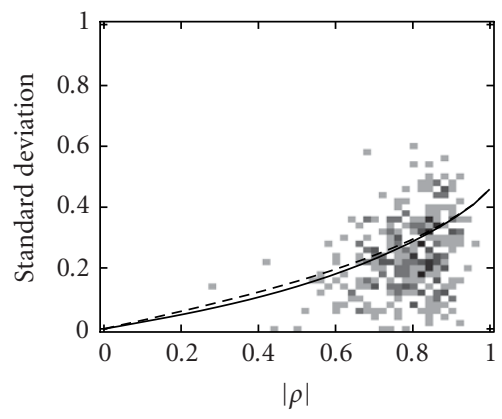

(b)

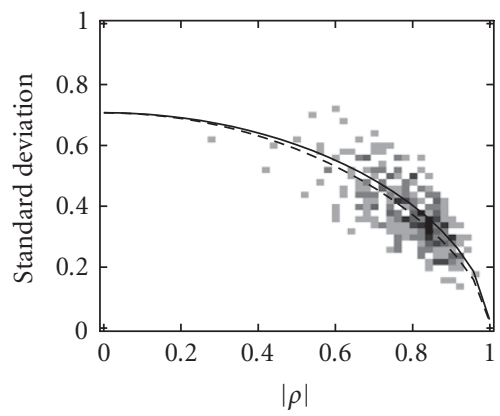

(e)

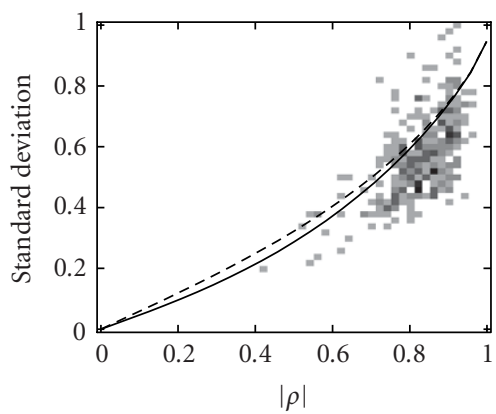

(h)

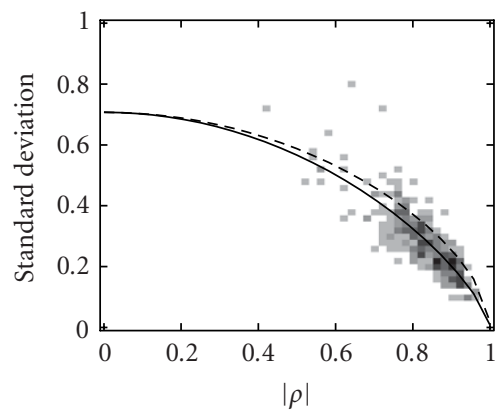

(k)

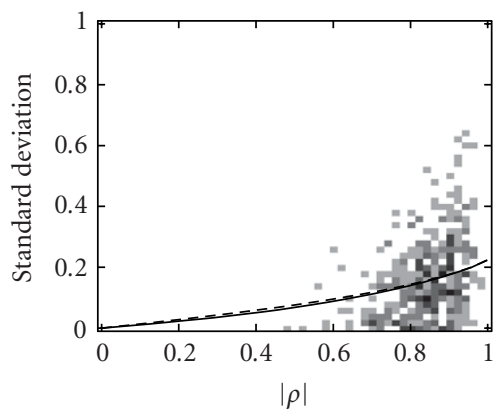

(c)

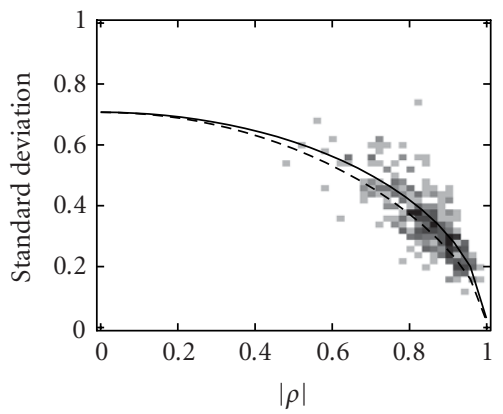

(f)

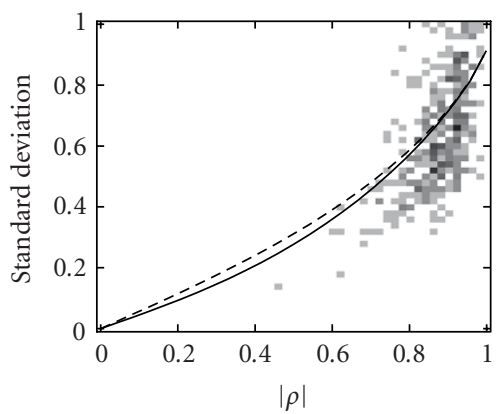

(i)

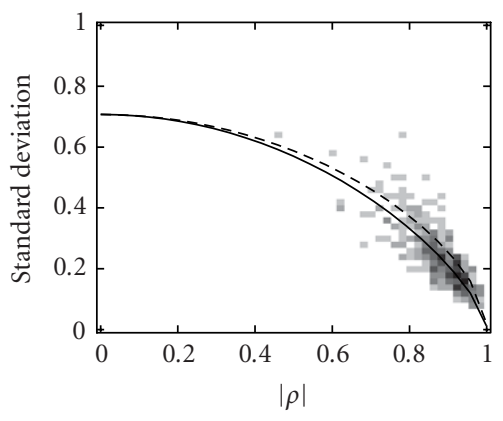

(1)

FIGURE 8: Normalized $(\psi=1)$ standard deviation curves for the multiplicative and additive speckle noise terms of the terms $\mathfrak{R}\left\{S_{h h 1} S_{v v 1}^{*}\right\}$ (PolSAR) and $\mathfrak{R}\left\{S_{h h 1} S_{v v 2}^{*}\right\}$ (PolInSAR). The first and third rows correspond to the multiplicative speckle term whereas the second and fourth rows correspond to the additive speckle term. Columns correspond to the three homogeneous areas analyzed, whose average complex correlation coefficients are indicated. (a), (d) $\rho_{h h 1 v v 2}=0.806 \exp (-j 0.277)$. (b), (e) $\rho_{h h 1 v v 2}=0.780 \exp (-j 1.092)$. (c), (f) $\rho_{h h 1 v v 2}=0.834 \exp (-j 1.798)$. (g), (j) $\rho_{h h 1 v v 1}=0.861 \exp (j 0.324)$. (h), (k) $\rho_{h h 1 v v 1}=0.812 \exp (j 0.316) .(\mathrm{i}),(\mathrm{l}) \rho_{h h 1 v v 1}=0.868 \exp (j 0.410)$. 


\section{REFERENCES}

[1] J. C. Curlander and R. N. McDonough, Synthetic Aperture Radar: Systems and Signal Processing, John Wiley \& Sons, New York, NY, USA, 1991.

[2] F. M. Henderson and A. J. Lewis, Principles and Applications of Imaging Radar, vol. 2, John Wiley \& Sons, New York, NY, USA, 3rd edition, 1998.

[3] R. Bamler and P. Hartl, "Synthetic aperture radar interferometry," Inverse Problems, vol. 14, no. 4, pp. R1-R54, 1998.

[4] D. Massonnet, M. Rossi, C. Carmona, et al., "The Displacement field of the landers earthquake mapped by radar interferometry," Nature, vol. 364, no. 6433, pp. 138-142, 1993.

[5] A. K. Gabriel, R. M. Goldstein, and H. A. Zebker, "Mapping small elevation changes over large areas: differential radar interferometry," Journal of Geophysical Research, vol. 94, no. B7, pp. 9183-9191, 1989.

[6] F. T. Ulaby and C. Elachi, Radar Polarimetry for Geoscience Applications, Artech House, Norwood, Mass, USA, 1990.

[7] I. Hajnsek, E. Pottier, and S. R. Cloude, "Inversion of surface parameters from polarimetric SAR," IEEE Trans. Geosci. Remote Sensing, vol. 41, no. 4, pp. 727-744, 2003.

[8] S. Allain, L. Ferro-Famil, and E. Pottier, "Surface parameter retrieval from polarimetric and multi-frequency SAR data," in Proc. IEEE International Geoscience and Remote Sensing Symposium (IGARSS '03), vol. 2, pp. 1417-1419, Toulouse, France, July 2003.

[9] S. R. Cloude and K. P. Papathanassiou, "Polarimetric SAR interferometry," IEEE Trans. Geosci. Remote Sensing, vol. 36, no. 5, pp. 1551-1565, 1998.

[10] A. Reigber and A. Moreira, "First demonstration of airborne SAR tomography using multibaseline L-band data," IEEE Trans. Geosci. Remote Sensing, vol. 38, no. 5, pp. 2142-2152, 2000.

[11] K. Sarabandi, “ $\Delta \mathrm{k}$-radar equivalent of interferometric SAR's: a theoretical study for determination of vegetation height," IEEE Trans. Geosci. Remote Sensing, vol. 35, no. 5, pp. 12671276, 1997.

[12] J. W. Goodman, "Some fundamental properties of speckle," Journal of the Optical Society of America, vol. 66, no. 11, pp. 1145-1149, 1976.

[13] J. W. Goodman, Statistical Optics, John Wiley \& Sons, New York, NY, USA, 1985.

[14] J.-S. Lee, "Speckle analysis and smoothing of synthetic aperture radar images," Computer Graphics and Image Processing, vol. 17 , no. 1 , pp. 24-32, 1981.

[15] A. Lopes, R. Touzi, and E. Nezry, "Adaptive speckle filters and scene heterogeneity," IEEE Trans. Geosci. Remote Sensing, vol. 28, no. 6, pp. 992-1000, 1990.

[16] C. Oliver and S. Quegan, Understanding Synthetic Aperture Radar Images, Artech House, Boston, Mass, USA, 1998.

[17] J.-S. Lee, M. R. Grunes, and G. de Grandi, "Polarimetric SAR speckle filtering and its implication for classification," IEEE Trans. Geosci. Remote Sensing, vol. 37, no. 5, pp. 2363-2373, 1999.

[18] S. R. Cloude and E. Pottier, "A review of target decomposition theorems in radar polarimetry," IEEE Trans. Geosci. Remote Sensing, vol. 34, no. 2, pp. 498-518, 1996.

[19] D. H. O. Bebbington, "Target vectors—spinorial concepts," in Proc. 2nd International Workshop on Radar Polarimetry, pp. 26-36, Nantes, France, September 1992.

[20] R. M. A. Azzam and N. M. Bashara, Ellipsometry and Polarized Light, Elsevier, Amsterdam, the Netherlands, 1997.
[21] J.-S. Lee, K. W. Hoppel, S. A. Mango, and A. R. Miller, "Intensity and phase statistics of multilook polarimetric and interferometric SAR imagery," IEEE Trans. Geosci. Remote Sensing, vol. 32, no. 5, pp. 1017-1028, 1994.

[22] R. J. A. Tough, D. Blacknell, and S. Quegan, "A statistical description of polarimetric and interferometric synthetic aperture radar data," Proceedings of the Royal Society of London. Series A, vol. 449, no. 1937, pp. 567-589, 1995.

[23] N. R. Goodman, "Statistical analysis based on a certain multivariate complex Gaussian distribution (an Introduction)," Annals of Mathematical Statistics, vol. 34, no. 1, pp. 152-177, 1963.

[24] R. J. Muirhead, Aspects of Multivariate Statistical Theory, John Wiley \& Sons, New York, NY, USA, 1982.

[25] G. Letac and H. Massam, "All invariant moments of the wishart distribution," Scandinavian Journal of Statistics, vol. 31, no. 2, pp. 295-318, 2004.

[26] K. Sarabandi, "Derivation of phase statistics of distributed targets from the mueller matrix," Radio Science, vol. 27, no. 5, pp. 553-560, 1992.

[27] J.-S. Lee, K. P. Papathanassiou, T. L. Ainsworth, M. R. Grunes, and A. Reigber, "A new technique for noise filtering of SAR interferometric phase images," IEEE Trans. Geosci. Remote Sensing, vol. 36, no. 5, pp. 1456-1465, 1998.

[28] C. López-Martínez, X. Fàbregas, and E. Pottier, "A new alternative for SAR imagery coherence estimation," in Proc. 5th European Conference on Synthetic Aperture Radar (EUSAR '04), Ulm, Germany, May 2004.

[29] C. López-Martínez and X. Fàbregas, "Modelling and reduction of SAR interferometric phase noise in the wavelet domain," IEEE Trans. Geosci. Remote Sensing, vol. 40, no. 12, pp. 2553-2566, 2002.

[30] C. López-Martínez and X. Fàbregas, "Polarimetric SAR speckle noise model," IEEE Trans. Geosci. Remote Sensing, vol. 41, no. 10, pp. 2232-2242, 2003.

[31] C. López-Martínez, X. Fàbregas, and E. Pottier, "Formulation and validation of a multidimensional SAR data speckle noise model," in Proc. 5th European Conference on Synthetic Aperture Radar (EUSAR '04), Ulm, Germany, May 2004.

Carlos López-Martínez received the M.S. degree in electrical engineering and the Ph.D. degree from the Technical University of Catalonia (UPC), Barcelona, Spain, in 1999 and 2003, respectively. In 1999, he joined the Signal Theory and Communications Department, UPC, where he developed his Ph.D. thesis, which focused on multidimensional speckle noise modeling and reduction. From October 2000 until March 2002, he was with the Frequency and Radar Systems Department (HR), German Aerospace Center (DLR), Oberpfaffenhofen. Since June 2003, he has been with the Image and Remote Sensing Group-SAPHIR Team, in the Institute of Electronics and Telecommunications of Rennes (IETR-CNRS UMR 6164). His research interests include SAR and multidimensional SAR, radar polarimetry, digital signal processing, estimation theory, and harmonic analysis. Mr. López-Martínez received the Student Prize Paper Award at the EUSAR 2002 Conference, Cologne, Germany. 
Xavier Fàbregas received the B.S. degree in physics from the Barcelona University, Barcelona, Spain, in 1988, and the Ph.D. degree in applied sciences from the Polytechnic University of Catalonia (UPC), Barcelona, Spain, in 1995. Since 1996, he has been an Associate Professor at UPC. His current research interests include SAR polarimetry, polarimetric retrieval algorithms, polarimetric calibration, and polarimetric SAR classification.

Eric Pottier received the M.S. and Ph.D. degrees in signal processing and telecommunication from the University of Rennes 1, respectively, in 1987 and 1990, and the Habilitation degree from the University of Nantes in 1998. Since 1999, he has been a Full Professor at the University of Rennes 1, France, where he is currently the Deputy Director of the Institute of Electronics and

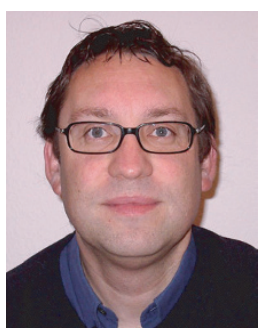
Telecommunications of Rennes (IETR-

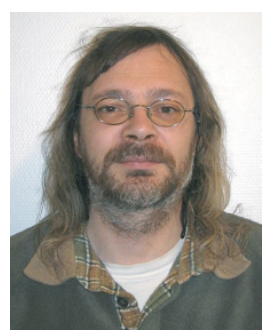
CNRS UMR 6164) and also Head of the Image and Remote Sensing Group-SAPHIR Team. His current activities of research and education are centered in the topics of analog electronics, microwave theory, and radar imaging with emphasis on radar polarimetry. His research covers a wide spectrum of areas from radar image processing (SAR, ISAR), polarimetric scattering modeling, and supervised/unsupervised polarimetric segmentation and classification to fundamentals and basic theory of polarimetry. Since 1989, he has supervised more than 50 research students to graduation (M.S. and Ph.D.) in radar polarimetry covering areas from theory to remote sensing applications. He has 6 publications in books, 25 papers in refereed journals, and more than 180 papers in conference and symposium proceedings. He has presented advanced courses and seminars on radar polarimetry to a wide range of organizations and events (DLR, NASDA, JRC, RESTEC, ISAP2000, IGARSS03, EUSAR04, NATO, PolInSAR05, IGARSS05). He received the Best Paper Award during EUSAR2000 for his research activities, coauthored with J. S. Lee (US Naval Research Laboratory), in the topic of polarimetric unsupervised segmentation of POL-SAR data. 